\title{
La traduction spécialisée à l'ère des nouvelles technologies : quel effet sur le texte de spécialité?
}

\section{The impact of new translation technologies on specialized texts}

\author{
Matthieu LeBlanc \\ Université de Moncton, Canada \\ matthieu.leblanc@umoncton.ca
}

\begin{abstract}
The introduction of translation technologies, especially translation memory software, has had a significant impact on both the translator's professional practice and the target text itself. Apart from the fact that he or she must translate in a non-linear fashion due to the design of translation memory systems, the translator is now called upon to increase output and, in many cases, recycle what has already been translated by others. As a result, the translator, used to having full control over his or her text, is in some regards losing control over the translation process, which brings him or her to reflect on the quality of the final product and, in turn, on the transformations the field of specialized translation is undergoing. In this paper, I will present the results of an important ethnographic study conducted in three Canadian translation environments. I will focus mostly on the effects translation technologies and newly implemented practices have had on the quality of specialized texts destined for the Canadian market, where most of the specialized texts produced in French are in fact translations. Special attention will be given to the comments made by specialized translators during semi-directed interviews.
\end{abstract}

Keywords: Specialized translation; translation technologies; ethnography; Canada

\section{INTRODUCTION}

Il est de fait que l'avènement des outils d'aide à la traduction a dans bien des cas eu des répercussions tant sur les pratiques professionnelles du traducteur que sur le texte d'arrivée lui-même (Mossop, 2006; Pym, 2011). Il suffit de penser aux mémoires de traduction, largement répandues dans le monde de la traduction professionnelle, qui, de par leur conception, ont eu des répercussions importantes sur la manière dont les traducteurs abordent le texte de départ - par 
segments, souvent phrase par phrase - et contribué au recyclage du « déjà traduit ». Si les conditions d'utilisation des mémoires de traduction varient certes - et parfois grandement - d'un milieu à l'autre, il n'en demeure pas moins que leur implantation a, dans certains cas précis, entraîné une perte de maîtrise sur le processus (Taravella et Villeneuve, 2011), dans la mesure où le traducteur, autrefois maitre de son texte, se voit de plus en plus contraint d'aborder le texte par segments, souvent de façon discontinue (Garcia, 2009; LeBlanc, 2013). Cette façon de faire n'est pas sans soulever des questions sur les conditions d'exercice de la profession, en pleine mutation depuis déjà bon nombre d'années, et, surtout, les conséquences sur le produit fini, c'est-à-dire le texte d'arrivée.

Dans cet article, nous nous intéresserons aux traducteurs de textes spécialisés en insistant sur l'effet des mémoires de traduction sur le processus de traduction et en particulier sur la qualité du produit fini. Nous nous inspirerons de données qualitatives recueillies sur le terrain, c'est-à-dire en milieu de travail, auprès de traducteurs spécialisés. Notre étude portera plus précisément sur le Canada et sur la traduction vers le français, langue de la minorité linguistique, en insistant sur le rôle de la traduction, et ipso facto des traducteurs, au sein de la société réceptrice. Nous nous intéresserons plus précisément à la traduction spécialisée, sachant qu'il s'agit d'un " métier en pleine mutation, du fait de la mondialisation des marchés qui multiplie et diversifie les besoins, et de l'évolution de la communication écrite liée à l'explosion des technologies de l'information, qui transforme une activité pensée comme traditionnelle en une diversité de compétences à la fois pointues et fortement intégrées. Contrairement à ce que l'on pourrait penser, malgré le développement de la traduction automatique et de la traduction assistée par ordinateur, on n'a probablement jamais tant eu besoin de traducteurs spécialisés qu'aujourd'hui. Mais la traduction a perdu son autonomie, elle n'a jamais été aussi dépendante de facteurs autres que linguistiques - principalement l'évolution des outils et de la conjoncture économique » (Lavault-Olléon, 2006 : ix-x; c'est nous qui soulignons).

Notre article se divisera en sept parties. Dans un premier temps, nous donnerons des précisions sur la traduction professionnelle telle qu'elle se pratique au Canada (origines, législation linguistique, "directionnalité » de la traduction), puis nous discuterons du rôle de la traduction vers le français dans un contexte où cette langue est minoritaire. Ensuite, nous fournirons des détails sur l'étude que nous avons menée auprès de traducteurs spécialisés dans trois services et entreprises de traduction (données sur les services et entreprises, méthodologie, etc.). La majeure partie de notre article sera consacrée à la présentation et à l'analyse d'une portion des données qui y ont été recueillies. Il sera d'abord question de l'effet des mémoires de traduction sur le procédé de 
traduction et sur les exigences de productivité. Par exemple, en quoi 1'introduction de cet outil a-t-elle bouleversé les pratiques administratives et commerciales? Nous nous pencherons ensuite et surtout sur l'effet de ces transformations sur la qualité des traductions spécialisées, dans un premier temps, et sur le rôle de la traduction spécialisée au sein de la société réceptrice, dans un deuxième temps. S'ensuivra la conclusion.

\section{LA TRADUCTION PROFESSIONNELLE AU CANADA}

Fort de son bilinguisme officiel anglais-français, le Canada est un pays où l'on pratique la traduction spécialisée depuis déjà bon nombre d'années et où la traduction - tout comme la terminologie et l'interprétation - jouit d'une réputation certaine à l'échelle mondiale. Les activités de traduction professionnelle se sont intensifiées de façon considérable à la suite de la promulgation de la Loi sur les langues officielles du Canada en 1969, loi qui fera de l'anglais et du français les langues officielles du pays, et qui par conséquent leur accordera un statut et des droits et privilèges égaux quant à leur usage dans les institutions du Parlement et du gouvernement du Canada. Cette même année, la province canadienne du Nouveau-Brunswick déclarait de son côté l'anglais et le français langues officielles en adoptant la Loi sur les langues officielles du NouveauBrunswick. S'est ensuivie en 1977 l'adoption par le Québec de la Charte de la langue française, laquelle fera du français la seule langue officielle de cette province. Constatant un essor de la demande de traducteurs, notamment dans les combinaisons anglais-français, les universités canadiennes ont lancé, en vagues successives à partir des années 70, des programmes de formation en traduction professionnelle, l'objectif étant de former des spécialistes compétents pour occuper les nombreux postes qui allaient voir le jour à la fois dans les fonctions publiques - notamment le gouvernement canadien, par le truchement du Bureau de la traduction, mais aussi dans certaines administrations provinciales telles que celles du Nouveau-Brunswick et de l'Ontario -, dans les cabinets privés et les agences de traduction, puis dans les services internes d'entreprises privées.

Malgré la présence de nombreuses communautés linguistiques au Canada, notamment dans les grands centres urbains, la traduction, comme activité, se pratique essentiellement entre l'anglais et le français, les langues officielles. S'il existe certes une activité de traduction dans les langues non officielles (arabe, mandarin, espagnol, p. ex.), elles occupent toutefois une place beaucoup moins grande que la traduction dans les langues officielles. Dans le résumé de l'Enquête auprès de l'industrie canadienne de la traduction publié en 1999, on indique que la traduction des langues officielles représente entre $75 \%$ et $81 \%$ des 
activités de traduction au pays (Comité sectoriel de l'industrie de la traduction canadienne, 1999: 7). On y ajoute que la plupart des traducteurs travaillent dans les grandes régions urbaines du Canada, notamment au Québec et en Ontario (1999 : 4), et que les principaux employeurs sont les cabinets de traduction, les gouvernements et les entreprises privées (1999). Qui plus est, bon nombre de traducteurs travaillent à leur propre compte.

Enfin, selon une analyse menée pour le compte du Bureau de la traduction du Canada, «[b]ien que le Canada ne compte que 0,5\% de la population mondiale, il représente environ $10 \%$ du marché mondial de la traduction, dont la valeur [en 2007] est de 31 milliards de dollars américains » (PricewaterhouseCoopers, 2010: 10). Ces chiffres illustrent bien le poids qu'occupe le Canada dans le marché mondial de la traduction et, surtout, l'incidence du bilinguisme institutionnel sur les activités de traduction dans ce pays.

\section{LE RÔLE DE LA TRADUCTION DANS L'AMÉNAGEMENT DU FRANÇAIS AU CANADA}

Au recensement de 2011, les Canadiens ayant déclaré avoir l'anglais comme langue maternelle, ou « anglophones », représentaient $57,8 \%$ de la population du pays et constituent ainsi le groupe linguistique majoritaire. Les francophones, c'est-à-dire ceux qui ont le français comme langue maternelle, viennent en deuxième place, représentant $22,3 \%$ de la population canadienne. Bien qu'ils soient dispersés un peu partout au pays, les francophones sont néanmoins concentrés dans la province du Québec, où ils sont majoritaires, ainsi que dans les provinces limitrophes de l'Ontario et du Nouveau-Brunswick, où ils demeurent minoritaires.

Malgré l'importance démographique des francophones au Canada - ils représentent en tout près de 7000000 de locuteurs -, on traduit tout de même beaucoup moins à partir $d u$ français que vers le français. Il est difficile d'obtenir des chiffres précis sur la proportion du volume de traduction qui s'effectue à partir du français et vers le français à l'échelle du pays, mais à s'en tenir aux données du Bureau de la traduction du Canada, baromètre de l'offre et de la demande, force est de constater que la majorité des textes issus de la fonction publique canadienne sont rédigés en anglais (voir Mossop, 1990). Il en va de même dans les secteurs du commerce et de l'industrie, où l'anglais domine non seulement comme langue de travail, mais aussi comme langue principale de rédaction. Bien sûr, au Québec, grâce aux dispositions de la Charte de la langue française, on rédige aussi en français, ce qui veut dire qu'on traduit forcément vers l'anglais, mais toute proportion gardée, pour l'ensemble du Canada, c'est 
majoritairement dans le sens anglais-français que se fait la traduction. Comme l'affirme Mareschal, «[1]a démographie canadienne, qui est constituée grosso modo de $70 \%$ d'anglophones et de $30 \%$ de francophones, a pour effet que les communications se font d'abord et principalement en anglais et que le gros de la demande de traduction se situe vers le français » $(2005: 252)$.

Sur le plan régional, la situation est comparable. Au Nouveau-Brunswick, seule province officiellement bilingue au Canada, plus de $88 \%$ des textes traduits par le Bureau de traduction, organisme à part entière du gouvernement néo-brunswickois, sont de l'anglais vers le français (Commissariat aux langues officielles du Nouveau-Brunswick, 2013 : 20), et cela même si les francophones représentent le tiers de la population de cette province. La plupart des communications, qu'elles émanent du secteur public ou du secteur privé, se font d'abord et avant tout en anglais pour ensuite être traduites vers le français.

C'est ainsi que, comme l'explique Brisset (1991), la traduction vers le français, au Canada, n'a pas qu'une simple valeur utilitaire; elle constitue un réel moteur de francisation en ce qu'elle permet au français de se faire voir dans des domaines où, sans la traduction, seul l'anglais serait présent. Par la diffusion d'un lexique et d'une terminologie française, la traduction est donc considérée comme un moyen de « faire contrepoids à la concurrence de la langue anglaise (...) » (Corbeil, 2007 : 92). La traduction a dans une large mesure une fonction non seulement utilitaire mais également dissimilatrice, puisque l'on s'en sert pour «promouvoir l'usage et la qualité du français au sein de la population francophone », et cela partout au Canada (Juhel, 1982: 60). De là la fonction éminemment sociolinguistique de la traduction dans la francophonie canadienne.

\section{L'ÉTUDE EN MILIEU DE TRAVAIL}

Notre étude avait pour premier objectif de mieux cerner le rapport que les traducteurs professionnels entretiennent avec les outils qu'ils utilisent au quotidien. Menée dans trois entreprises et services de traduction au Canada, l'étude était de nature entièrement qualitative, l'accent ayant été mis sur les traducteurs eux-mêmes, puis sur le rôle des technologies langagières dans le processus de traduction et les conditions d'exercice de la profession. Au total, nous avons passé plus de 300 heures au sein de trois différents services ou entreprises de traduction, sur une période s'étalant sur à peu près trois mois. Tous de taille moyenne, les services et entreprises à l'étude comptaient entre 22 et 80 employés et proposaient des services de traduction générale et de traduction spécialisée dans les langues officielles, presque exclusivement de l'anglais vers le français. Généralistes de formation, les traducteurs employés 
par ces services et entreprises se livraient tous à la traduction de textes généraux et spécialisés dans une multitude de domaines: informatique, médecine, administration publique, sciences naturelles, droit, économie, gestion, etc. Il importe de préciser que les textes traduits par les services et entreprises à l'étude étaient destinés à un public canadien - surtout canadien-français - et que les demandeurs d'ouvrage étaient sauf exception tous canadiens. Enfin, les services et entreprises comptaient dans leurs rangs des traducteurs débutants, intermédiaires et chevronnés de toutes les tranches d'âge. Tous avaient tous fait des études universitaires en traduction ou encore dans un domaine connexe (linguistique, p.ex.), et la grande majorité d'entre eux étaient originaires du Canada.

Concernant l'utilisation des technologies langagières, les services et entreprises de traduction à l'étude proposaient à leurs traducteurs des outils à la fine pointe de la technologie. Autrement dit, il s'agissait de milieux très « technologisés », en ce sens que les traducteurs se servaient tous d'une panoplie d'outils d'aide à la traduction (dictionnaires électroniques, bases de données terminologiques, concordanciers, etc.). L'outil autour duquel s'organisait le travail était cependant le gestionnaire de mémoire de traduction, que tous les employés, sans exception, devaient maîtriser. Dans l'un des services en question, l'on avait commencé à introduire la traduction automatique statistique, à titre d'essai.

Sur le plan méthodologique, notre démarche était, comme nous l'avons précisé plus haut, éminemment qualitative. Aux activités d'observation générale sont venues s'ajouter des séances d'observation des traducteurs à l'œuvre, c'està-dire à leur poste de travail, lesquelles nous ont permis de voir comment les traducteurs se servent des outils à leur disposition, notamment les mémoires de traduction, puis d'interroger ces derniers sur leur interaction avec les outils. En tout, nous avons mené 21 séances d'observation auxquelles se sont ajoutés 52 entretiens semi-dirigés auprès de traducteurs et réviseurs, en grande partie, puis de quelques gestionnaires. Les entretiens - dont la longueur variait entre 25 et 75 minutes - nous ont permis d'établir le profil de chacun des traducteurs (âge, expérience professionnelle, etc.) et d'obtenir des précisions sur leurs méthodes de travail, les types de textes qu'ils étaient appelés à traduire (domaines de spécialité), leur utilisation des outils (informatiques et traditionnels) et le rapport qu'ils entretiennent avec les outils et les textes. Très riches, les entretiens, jumelés aux activités d'observation, nous ont permis de mieux saisir les pratiques à l'œuvre dans les milieux à l'étude, les transformations qui s'opèrent, puis les défis qui se posent. Enfin, les services et entreprises de traduction à l'étude nous ont fourni des données quantitatives et qualitatives (mode de gestion, exigences de productivité, implantation des outils, 
perfectionnement des traducteurs, etc.) qui nous ont aidé à mieux cerner les pratiques administratives et commerciales à l'œuvre.

$\mathrm{Si}$ nous avions au premier abord prévu nous concentrer sur les rapports qu'entretiennent les traducteurs avec les outils d'aide à la traduction, notre recherche sur le terrain nous a aussi permis de récolter des données sur de multiples autres aspects de la pratique professionnelle, en particulier le lien entre la formation des traducteurs et la réalité professionnelle ainsi que l'effet de certains outils sur la qualité globale des traductions. En effet, bien des traducteurs professionnels se sont prononcés tant sur les transformations que subit la profession de traducteur au Canada - notamment sous l'effet du resserrement des exigences de productivité lié à l'implantation de certains outils d'aide à la traduction - que sur l'effet de ces transformations sur la société réceptrice, en l'occurrence les francophones, qui sont, comme nous l'avons précisé, de grands consommateurs de traduction. Dans bien des cas, la quasi-totalité des textes qui parviennent aux yeux des francophones, notamment ceux qui vivent en situation minoritaire à l'extérieur du Québec, sont des traductions de l'anglais. C'est ainsi que nous avons choisi, pour les besoins du présent article, d'analyser en profondeur ces données fort probantes, dans la mesure où, dépassant le cadre des simples technologies, elles touchent au rôle social du traducteur et, par ricochet, de la traduction spécialisée dans la francophonie canadienne.

\section{LES TRANSFORMATIONS ENTRAÎNÉES PAR L'INTÉGRATION DES MÉMOIRES DE TRADUCTION}

Dans un article que nous avons publié en 2013 - dont nous résumons les grandes lignes dans un souci de contextualisation -, nous avons énuméré les avantages et les inconvénients des mémoires de traduction aux yeux des traducteurs qui travaillent dans les trois entreprises et services de traduction qui feront l'objet du présent article (LeBlanc, 2013). Si dans l'ensemble les traducteurs y voient certains avantages - notamment l'accroissement de la productivité dans le cas de textes répétitifs -, ils y voient aussi un certain nombre de désavantages. Outre qu'elles entraînent une segmentation non naturelle du texte et modifient ainsi le rapport au texte (voir LeBlanc, 2015 [à paraître] à ce sujet), les mémoires de traduction, employées dans l'unique but d'accroître la productivité et la rentabilité, auraient pour effet d'émousser la créativité des traducteurs, d'encourager la passivité, d'entraîner une certaine dépendance aux outils, de nuire aux réflexes naturels, puis de soumettre indûment les traducteurs aux traductions existantes (LeBlanc, 2013). 
Ce que déplorent le plus les traducteurs, cependant, c'est que l'implantation de ces outils a fini par avoir un effet sur les pratiques commerciales et administratives des services et entreprises en question (LeBlanc, 2013). Plus précisément, les mémoires de traduction ont permis aux entreprises et services d'instaurer de nouvelles manières de calculer la productivité en tenant compte du « déjà traduit », plus précisément les correspondances exactes, parfaites (100\%) ou partielles $(75 \%)$, puis en obligeant du coup les traducteurs à recycler ce qui figure dans la mémoire de traduction. Ce recyclage forcé, jumelé à l'accroissement des exigences de productivité individuelle, constitue un revirement assez brusque pour la plupart des traducteurs des services et entreprises à l'étude (LeBlanc, 2013). Ce sont somme toute des pratiques qui, d'une part, les ont obligés à accélérer leur rythme de travail et à compter de plus en plus sur le « déjà traduit». Enfin, ces changements - draconiens dans certains cas - ont amené de nombreux traducteurs à s'interroger sur l'effet de ces nouvelles pratiques sur le texte d'arrivée.

Dans les sections qui suivent, nous nous examinerons en détail la manière dont ces transformations se sont répercutées sur la qualité du produit fini, toujours aux yeux des traducteurs. Nous aborderons ensuite le rôle du traducteur spécialisé et de la traduction spécialisée dans la société réceptrice, éléments qui ressortent de façon récurrente de nos données.

\section{L'EFFET DES TRANSFORMATIONS TECHNOLOGIQUES SUR LA QUALITÉ DES TRADUCTIONS SPÉCIALISÉES}

Interrogés sur les transformations qui ont marqué leur profession au cours des dix dernières années, les traducteurs ont pour la plupart insisté sur l'effet des nouvelles pratiques administratives et commerciales sur la qualité des traductions qu'ils produisent au quotidien. Appelés à maintenir un rythme de productivité toujours croissant, et ipso facto à recycler de plus en plus les traductions existantes, les traducteurs affirment dans un premier temps avoir de moins en moins l'occasion de se documenter avant d'entreprendre la traduction de leurs textes. Comme le signale cette traductrice, il est plutôt rare, aujourd'hui, que les traducteurs puissent prendre le temps de se documenter convenablement, et cela même si cette pratique a été courante et fortement encouragée pendant très longtemps dans le milieu au sein duquel elle travaille :

TR05A: Moi, je traduis dans deux domaines, essentiellement, l'agriculture et les ressources naturelles. Ce sont mes deux domaines de spécialité.

Matthieu : D'accord. 
TR05A : Il n'y a pas si longtemps, lorsque les exigences de productivité étaient moins élevées, je passais parfois beaucoup de temps à me documenter. (...) Dans la bibliothèque du service, on a toutes sortes d'ouvrages et même des revues dans ces domaines de spécialité. C'était justement dans ces sources que je me documentais, que je trouvais souvent la réponse à mes questions. Aujourd'hui, il nous est impossible de trouver le temps de faire ça. C'est un luxe.

Matthieu : C'est un luxe de pouvoir se documenter?

TR05A : Oui, tout à fait, on n'arrive rarement à le faire. Ça n'a pas toujours été comme ça.

Cette difficulté qu'ont les traducteurs à se documenter - difficulté intimement liée aux contraintes de temps et non à la disponibilité de la documentation - les amène à réfléchir sur rôle clé des ressources documentaires et des textes parallèles en traduction spécialisée, comme nous l'explique ce traducteur :

TR13A : À la base, la traduction d'un texte spécialisé suppose un travail préalable de documentation. Il faut savoir de quoi on parle. Je ne vous apprends rien. $C$ 'est ce qu'on nous a d'ailleurs enseigné à l'université et ce que j'ai fait dans ma pratique professionnelle depuis mes tout débuts en traduction. Ou presque toujours fait.

Matthieu : Oui, oui.

TR13A : Aujourd'hui, on ne semble plus valoriser ce travail de documentation, qui est pourtant essentiel. L'ironie, c'est qu'on a plus que jamais accès à toutes sortes de sources documentaires, en ligne surtout, et même à des textes parallèles, mais on n'a plus le temps de les consulter. C'est vraiment dommage, c'est une perte.

Matthieu : Oui, et que faites-vous dans ces cas-là?

TR13A: En fait, c'est la mémoire de traduction qui devient notre référence, notre seule référence, par la force des choses. Et bien évidemment, la mémoire de traduction, ce n'est pas une ressource documentaire spécialisée. Ce sont des traductions recyclées.

Matthieu : Oui, ok, je comprends.

TR13A: C'est utile, oui, c'est pratique, mais ce ne pas là où on trouve des renseignements sur le domaine, des notions, des exemples de tournures et formulations propres à un domaine de spécialité. Ce sont dans les textes spécialisés rédigés en français qu'on trouve ça. Se limiter aux mémoires de traduction, ce n'est pas rendre service, rendre justice au texte, au lecteur.

Matthieu : Non?

TR13A : Non, c'est du réchauffé, du recyclé, du tout cuit. On ne sait pas qui l'a traduit [le texte ou les segments répertoriés dans la mémoire] ou encore s'il s'agit d'un traducteur spécialisé dans le domaine en question. 
Ce que l'on constate dans les trois services et entreprises à l'étude, c'est que la mémoire est en quelque sorte devenue, sous l'effet du resserrement des exigences de productivité, la première, voire la seule, source documentaire pour les traducteurs. L'époque où le traducteur spécialisé pouvait consulter des sources documentaires dans le but d'approfondir sa connaissance d'un domaine et se familiariser avec les modèles de rédaction dans la langue d'arrivée semble révolue, du moins dans les milieux à l'étude. D'aucuns s'interrogent ainsi sur l'effet de cet état de choses sur la qualité du produit fini, le texte d'arrivée. Témoin le commentaire émis par la traductrice suivante :

Matthieu : Et au juste, c'est quoi cette pression à laquelle vous faites allusion?

TR12B : C'est cette pression vraiment accrue de traduire avec rapidité à l'aide des outils. Et ça a sans doute un effet sur la qualité du texte. C'est indéniable. Tous mes collègues en conviendront, j'en suis certain.

Matthieu : Vous n'êtes pas la première à le mentionner, en fait.

TR12B: C'est qu'en traduisant phrase par phrase, dans des petites cases, et en reprenant tout cru ce qu'on a déjà proposé dans une traduction antérieure, ou encore ce que quelqu'un d'autre a proposé, on finit par ne plus penser en français.

Matthieu : Que voulez-vous dire au juste?

TR12B : Prenons l'exemple du texte que je vous ai montré plus tôt ce matin, le texte médical. Je traduis dans ce domaine, entre autres, depuis quand même bon nombre d'années. Je m'y connais bien, heureusement. Mais cette aisance, je l'ai acquise au fil des années, par mes lectures, les recherches quand même approfondies que j'ai pu faire dans les domaines en question. Les pauvres jeunes [les débutants], eux, ils n'ont pas eu cette chance. Ils n'arrivent pas à se documenter, à s'imprégner des tournures en langue d'arrivée, à voir comment on exprime telle ou telle réalité en français. Ils n'ont que la mémoire de traduction et n'ont pas le temps de se documenter. La qualité en souffre beaucoup, et c'est la lutte constante entre qualité et quantité.

Matthieu : Ok. Est-ce que c'est à cette tension ou à ce malaise que vous faisiez allusion plus tôt?

TR12B : Oui, tout à fait.

Cette tension entre quantité et qualité, les traducteurs disent la ressentir de plus en plus sous l'effet des nouvelles pratiques administratives et commerciales adoptées par suite de l'intégration des mémoires de traduction. Le mot qualité revient systématiquement dans les entretiens et dans les discussions, et l'on comprend qu'il s'agit d'une réelle préoccupation pour bien des traducteurs. On sent de plus que les nouvelles conditions d'exercice sont en rupture avec celles qu'ont connues les traducteurs d'expérience lorsqu'ils étaient en début de carrière. C'est justement ce qu'affirme cette traductrice-réviseure : 
TR11C: Lorsque j'ai été embauchée il y a neuf ans, c'était très, très différent. Mes réviseurs et mes superviseurs insistaient sur la qualité, comme c'était le cas à l'université. C'était, disons, le mot d'ordre.

Matthieu : Et tu me dis que t'as noté des changements?

TR11C: Ah oui. Je ne dis pas qu'on n'accordait pas à cette époque une certaine importance à la vitesse d'exécution, qu'on n'avait pas d'objectifs à atteindre. Ce n'est pas ça. On avait bien sûr des cibles de productivité. Mais en fin de compte, on cherchait une traduction de qualité, qui répondait aux exigences des lecteurs, des destinataires.

Matthieu : $O k$.

TR11C: On insistait, comment dire, sur la lisibilité du texte en français. Il fallait se documenter. Là, c'est plus difficile. Beaucoup plus difficile. (...) Je m'interroge beaucoup sur la qualité, car tout est fait rapidement. Je remets tout en question.

\section{LE RÔLE DE LA TRADUCTION SPÉCIALISÉE AU SEIN DE LA SOCIÉTÉ RÉCEPTRICE}

Ces nombreuses interrogations sur la qualité des traductions spécialisées ne sont pas indissociables d'une part du terrain d'implantation des services et entreprises à l'étude et d'autre part de la société réceptrice des traductions, en l'occurrence les francophones du Canada.

Ce qui ressort souvent des entretiens, notamment chez les traducteursréviseurs intermédiaires et chevronnés, c'est la crainte de produire des traductions qui, collant de trop près à l'anglais, manquent d'idiomaticité et de transparence. L'expression «calque de l'anglais » (ou «calqué de l'anglais ») est fréquemment évoquée. Par «calque », les traducteurs ne font par forcément référence à une interférence ponctuelle ou à un anglicisme syntaxique, mais plutôt à l'ensemble du texte, à sa structure globale, au modèle qui est reproduit. Nombreux sont les traducteurs qui, faute de temps, disent ne plus pouvoir produire des textes semblables à ceux qui sont produits spontanément en langue d'arrivée. Autrement dit, ils sont de plus en plus contraints de calquer le modèle de l'anglais, comme l'explique ce réviseur :

TR14A : J'ai de plus en plus l'impression, et je pense avoir raison, que le texte que nous livrons, c'est-à-dire notre produit fini, colle de beaucoup plus près à l'anglais, au modèle de l'anglais, que par le passé.

Matthieu : Ah ok. Et comment se fait-il? Comment vous l'expliquer?

TR14A: Ce n'est pas que les textes renferment des gros anglicismes, des calques, par exemple, mais c'est la structure du texte, son organisation. On ne s'éloigne plus du modèle anglais.

Matthieu : Dans la présentation? 
TR14A : Oui, oui, et dans son organisation. C'est comme s'il fallait à tout prix avoir un modèle, un seul, le même, pour l'anglais et le français. Dans ce texte ici [il me montre le texte], tu ne trouveras pas d'erreurs majeures ou de calques. Mais ce qui est calqué, ou imité, c'est la structure globale du texte anglais. C'est un calque de modèle, si vous voulez. En français, normalement, on ne commencerait jamais un texte en présentant cette information-là au tout début. On la présenterait plus loin dans le texte. C'est ce qu'on faisait avant, on s'inspirait du modèle français.

Matthieu : C'est une consigne qu'on vous donne?

TR14A : Indirectement. Indirectement. La mémoire [de traduction], comme tu le sais, aime bien que tout soit parfaitement aligné. Dans certains cas, ça va. Pour les notices techniques, par exemple, c'est universel, pareil dans toutes les langues. Mais c'est pas vrai dans tous les cas, dans tous les domaines de spécialité.

$\mathrm{Au}$ dire du traducteur précité, on observe une tendance à s'éloigner des modèles textuels en langue d'arrivée, d'où la référence à l'expression « calque de modèle », soit un texte qui ne tiendrait pas compte des pratiques rédactionnelles du français, des schèmes de pensée, bref qui reflète le modèle anglais.

Il est intéressant de noter que, dans l'extrait ci-dessus, le traducteur est fort conscient du fait que, pour certains types de textes (techniques et scientifiques, notamment), la distance «culturelle » est minime, alors que pour d'autres, elle est plus grande et exige donc que les traducteurs soient en mesure de réorganiser le texte d'arrivée, au besoin. Les contraintes de temps, jumelées à la segmentation forcée du texte, ne permettent plus aux traducteurs de modifier la structure du texte d'arrivée dans le dessein de le rendre plus conforme aux habitudes et aux attentes des lecteurs francophones dans le domaine de spécialité en question.

Parallèlement aux réflexions sur l'organisation du texte en langue d'arrivée, sur sa structure globale, les traducteurs s'interrogent sur la communauté réceptrice des traductions. Dans le commentaire qui suit, l'une des traductrices s'interroge sur son propre travail et plus largement sur le rôle de la traduction en milieu minoritaire francophone, notamment dans les régions situées à l'extérieur du Québec. Sachant que les francophones sont de grands consommateurs de traduction, elle déplore le fait que l'on ne se préoccupe pas davantage du rôle social de la traduction et de l'effet des traductions-calques sur la société réceptrice. Le commentaire ci-dessous illustre avec éloquence ses préoccupations :

TR02B: Ça [le fait que les francophones soient de grands consommateurs de traduction] nous amène, en tout cas ça m'amène, moi, à réfléchir sur ce que je fais, la nature de la traduction, plutôt le rôle de la traduction. C'est clair. 
Matthieu : Le rôle de la traduction à son sens large?

TR02B: Oui, justement. La plupart des textes officiels, des textes spécialisés, des textes techniques qu'on lit ici sont des traductions. Au Québec, un peu moins, mais quand même une bonne proportion, qu'on le veuille ou non.

Matthieu : Oui, exactement.

TR02B : Il faut donc prendre un peu de recul, se poser des questions. On traduit au jour le jour sans trop parfois penser à ceux qui liront nos traductions. À force d'imiter l'anglais, de tout faire de plus en plus rapidement, on finit par offrir un produit de plus faible qualité, comment dire, de qualité moindre.

Matthieu : Que par le passé?

TR02B : Oui, bien sûr. Elle est finie l'époque où on pouvait peaufiner nos textes pour s'assurer qu'ils ressemblent à un texte qui avait été rédigé en français.

Matthieu : $O k$.

TR02B : Et c'est à se demander quel effet ça peut avoir, à la longue, sur les destinataires. Si tout ce qu'on consomme, en tant que francophones, sont des traductions, ne faudrait-il pas tout faire pour que ces traductions soient impeccables? Les traductions deviennent des modèles, en quelque sorte. Les gens s'en servent, s'en inspirent. On ne peut pas se permettre de les faire à moitié. Tu vois ce que je veux dire?

Matthieu : Oui. Donc selon toi, la qualité, c'est primordial, c'est ça?

TR02B : Oui. Sans ça, ça devient un copier-coller de l'anglais. Et quel effet est-ce que ça finit par avoir sur les locuteurs? Sur la communauté? Il faut y penser.

Dans la même veine, une traductrice chevronnée fait référence à ce tiraillement qu'elle ressent, à cette pression qui s'exerce constamment sur elle :

TR12C: (...) "Tiraillé » serait le mot juste, selon moi, parce qu'on est toujours tiraillés, en quelque sorte. On sait que la traduction joue un rôle important dans l'épanouissement de la communauté francophone au Canada. La plupart des textes spécialisés que je traduis sont destinés à ce public-là, et j'en suis pleinement conscient.

Matthieu : Revenons à ce tiraillement que vous mentionnez.

TR12C: C'est qu'on est tiraillés entre le désir, ou la pression plutôt, d'en faire toujours plus et la mission qu'on nous confie. Traduire pour un public, une minorité, qui dépend quand même beaucoup de la traduction pour son épanouissement, comme je le disais, c'est pas comme traduire pour n'importe quel public. Il y a une différence.

Matthieu : Dans la mission?

TR12C: C'est un peu ça. On traduit pour communiquer, véhiculer un message, mais si le véhicule est la traduction, comme c'est souvent le cas, notre responsabilité, en tant que traducteurs, est encore plus grande. Elle joue un rôle dans la société, qui n'est quand même pas négligeable.

Comme l'illustre ce dernier extrait, c'est le rôle social dévolu à la traduction (vers le français), et par ricochet le rôle sociolinguistique du traducteur en milieu 
francophone canadien, qui est à l'origine de ce tiraillement, de ce malaise, que ressent la traductrice précitée. Pour cette dernière, la traduction de textes spécialisée est investie d'une fonction sociale qui va bien au-delà de la simple fonction de communication qu'on associe souvent à l'acte de traduire. Le choix des termes «épanouissement» (de la communauté) et «responsabilité » témoignent du rôle social que les traducteurs attribuent à l'activité de traduction vers la langue de la minorité et de l'importance qu'ils accordent à la production de textes qui servent de modèles pour la société réceptrice.

\section{DISCUSSION ET CONCLUSION}

S'il est un élément qui ressort de notre recherche en milieu de travail, c'est la conscience, chez les traducteurs spécialisés, des transformations qui ont marqué leur profession au cours des quinze dernières années. À cette conscience des changements survenus se greffe une certaine réflexion tant sur l'évolution du rôle du traducteur dans la société que sur la place de la traduction spécialisée dans la société réceptrice, notamment dans des contextes de langue minoritaire. $\mathrm{Si}$, d'un côté, les traducteurs constatent que l'introduction de certains outils d'aide à la traduction a, dans une certaine mesure, entrâné des gains de productivité, voire une réduction du travail répétitif, d'un autre côté, ils constatent pleinement l'effet de ces outils sur les pratiques administratives et commerciales qui ont depuis lors été adoptées dans les entreprises et services au sein desquels ils travaillent.

Mais avant tout, ce sont les remarques sur la qualité des traductions qui ressortent le plus souvent des entretiens. Comme l'illustrent les passages reproduits plus haut, les traducteurs se rendent compte que les nouvelles conditions dans lesquelles ils sont appelés à travailler rendent difficile "l'adhésion aux normes et aux conventions de rédaction dans la langue-culture d'arrivée » (Scarpa, 2010 : 97). L'accroissement des exigences de productivité, intimement lié à l'introduction des mémoires de traduction, fait en sorte que ce sont non plus les sources documentaires spécialisées en langue d'arrivée ouvrages spécialisés, textes parallèles, etc. - qui servent d'inspiration et de modèles, mais plutôt les corpus de traductions incorporés aux mémoires de traduction. Autrement dit, les mémoires deviennent la première source de documentation et de référence, ce qui, comme l'ont fait remarquer bon nombre de traducteurs, a pour effet de nuire à la qualité du produit fini, dans la mesure où, à leurs yeux, une « traduction doit (...) parler la langue de ses destinataires et ressembler aux autres textes produits pour la même collectivité discursive spécialisée » (Scarpa, 2010 : 140) et non au modèle de l'anglais. 
Dans l'ensemble, les traducteurs, très sensibles aux aspects culturels de la traduction, insistent sur le rôle éminemment sociolinguistique de cette activité en situation de langue minoritaire, en l'occurrence la francophonie canadienne. Les nombreuses remarques et inquiétudes formulées à cet égard témoignent, elles aussi, d'une conscience aiguë de la place de l'anglais dans la société canadienne, d'une part, et du rôle de la traduction dans l'aménagement du français, d'autre part. Sans nier que le respect des délais et la vitesse d'exécution sont des contraintes avec lesquels tous les traducteurs doivent composer, les participants à notre étude sont néanmoins d'avis que, trop " encadrés 》 et trop astreints aux outils d'aide à la traduction, les traducteurs ne sont plus à même de se consacrer à l'essentiel de leur travail, à exercer leur "sensibilité interculturelle » (Katan, 2004 : 168-168; cité dans Scarpa, 2010 : 111), et voient de ce fait leur rôle réduit à celui de simples «transcodeurs» dont le travail est au bout du compte peu valorisé.

Ces questions, fort complexes, en appellent bien d'autres. Notre étude, qui se limite à trois milieux de travail canadiens, ne se veut bien évidemment pas exhaustive; néanmoins, il y a fort à parier que ce que nous avons observé dans ces milieux, notamment par rapport à l'effet des technologies sur les pratiques et sur le produit, s'observe aussi dans d'autres milieux et dans d'autres contextes. Il est donc impératif que les traductologues se penchent sur les transformations qui ont marqué le paysage de la traduction spécialisée ces dernières années et qu'ils tentent d'en mesurer les effets tant sur les textes de spécialité que sur la profession de traducteur spécialisé. Et surtout, il faut éviter que la traduction spécialisée demeure le parent pauvre de la traductologie. En se rendant sur le terrain de la traduction (Mossop, 2007) et en donnant la parole aux traducteurs spécialisés, il y a moyen de redonner à la traduction spécialisée ses lettres de noblesse et du coup de réduire l'écart - souvent perçu - entre la théorie et la pratique professionnelle.

Enfin, nous osons espérer que des études de ce genre, réalisées auprès de traducteurs en exercice, auront des implications tant pratiques que théoriques. $\mathrm{Si}$ les domaines de la traductologie et de la sociolinguistique peuvent bénéficier d'études ethnographiques en milieu de travail dans la mesure où elles permettent de mieux appréhender les rapports entre la traduction spécialisée et la société réceptrice, les milieux de la traduction - c'est-à-dire les entreprises privées, les services de traduction internes - et les traducteurs eux-mêmes, tant les salariés que les indépendants, sont eux aussi susceptibles d'être interpellés par les transformations observées dans la pratique professionnelle, lesquelles peuvent, au bout du compte, avoir une incidence sur leur satisfaction et leur autonomie professionnelles. 


\section{BIBLIOGRAPHIE}

Brisset, A. (1991). Le rôle de la traduction dans la société canadienne. L'Actualité terminologique, 24(1), 7-8.

Comité sectoriel de l'industrie canadienne de la traduction (1999). Enquête auprès de l'industrie canadienne de la traduction. Stratégie de développement des ressources humaines et d'exportation, Ottawa.

Corbeil, J.-C. (2007). Le rôle de la terminologie en aménagement linguistique : genèse et description de l'approche québécoise. Langages, 168(4), 92-105. DOI: 10.3917/lang.168.0092

Commissariat aux langues officielles du Nouveau-Brunswick (2013). Rapport annuel 2012-2013. Fredericton (Nouveau-Brunswick), Bureau du Commissaire aux langues officielles. http://www. languesofficielles.nb.ca/sites/default/files/imce/pdfs/FR/web_2_rapport_annuel_2012-2013.pdf

Garcia, I. (2009). Beyond Translation Memory: Computers and the Professional Translator. Journal of Specialized Translation, 12, 199-214. http://www.jostrans.org/issue12/art_gar cia.pdf

Juhel, D. (1982). Bilinguisme et traduction au Canada. Le rôle sociolinguistique du traducteur. Québec: Centre international de recherche sur le bilinguisme.

Katan, D. (2004). Translating Cultures. An Introduction for Translators, Interpreters and Mediators ( $2^{\mathrm{e}}$ éd.). Manchester: St. Jerome.

Lavault-Olléon, É. (2007). Introduction. In É. Lavault (Éd.), Traduction spécialisée : pratiques, théories, formations (p. ix-xii) Berne: Peter Lang.

LeBlanc, M. (2013). Translators on Translation Memory (TM). Results of an ethnographic study in three translation services and agencies. The International Journal for Translation \& Interpreting Research, 5(2), 1-13. DOI: ti.105202.2013.a01

LeBlanc, M. (2015 [à paraître]). Les mémoires de traduction et le rapport au texte : ce qu'en disent les traducteurs professionnels. TTR, 28(1).

Mareschal, G. (2005). L'enseignement de la traduction au Canada. Meta, 50(1), 250-262. DOI: $10.7202 / 010672 \mathrm{ar}$

Mossop, B. (2007). Empirical studies of revision: What we know and need to know. The Journal of Specialized Translation, 8, 6-20. http://www.jostrans.org/issue08/art_mossop.pdf

Mossop, B. (2006). Has computerization changed translation? Meta, 51(4), 787-793. DOI: 10.7202/014342ar

Mossop, B. (1990). Translating Institutions and 'Idiomatic' Translation. Meta, 35(2), 342-355. DOI: $10.7202 / 003675 \mathrm{ar}$

PricewaterhouseCoopers (2012). Analyse comparative du Bureau de la traduction. Rapport final. Toronto: PricewaterhouseCoopers.

Pym, A. (2011). What technology does to translating. The International Journal for Translation \& Interpreting Research, 3(1), 1-9. http://trans-int.org/index.php/transint/article/view/121/81

Scarpa, F. (2010). La traduction spécialisée. Une approche professionnelle à l'enseignement de la traduction. Ottawa: Presses de l'Université d'Ottawa. (Traduit et adapté de l'italien par Marco A. Fiola.)

Taravella, A.-M. et A.O. Villeneuve. Aspects humains des technologies langagières dans l'organisation. Tralogy (Session 2 - Le métier de traducteur). http://lodel.irevues.inist.fr/tralo gy/index.php?id=134 (consulté le 30 août 2015). 